\title{
Research and Application of Internet of Things
}

\author{
Jin-kuang Wang, Jing Zhang, Min-feng Yao, Shi-xing Li \\ and Jie-lun Li \\ Cisco School of Informatics, Guangdong University of Foreign Studies, \\ Guangzhou, China \\ Corresponding Authors: \{jkwang; yaomf\}@gdufs.edu.cn; ha_go@163.com; \\ \{578227896; lijielun.jay\}@qq.com
}

Received 1 October 2014; Accepted 20 April 2015;

Publication 1 June 2015

\begin{abstract}
This paper discusses the architectures, interfaces as well as behaviours of our model of an implemented intelligent device, a Robot. As a case study, the Robot has all the key features of the Internet of Intelligent Things (IoT): autonomous, mobile, and sensorial. By achieving the goal of having a computer or smart phone to access and control the Robot, we present an approach to control and monitor a remote object to move through the Internet.
\end{abstract}

Keywords: Internet of Things; Embedded System; Sensor; Measurement and Control Technology.

\section{Introduction}

Nowadays, about two billion people around the world use the Internet for browsing the World-Wide Web (WWW), sending and receiving emails, accessing multimedia contents and services, playing games, using social networking applications and many other tasks. While more and more people gain access to these global information and communication infrastructures,

Journal of Machine to Machine Communications, Vol. 1, 215-228.

doi: 10.13052/jmmc2246-137X.132

(c) 2015 River Publishers. All rights reserved. 
another big leap forward is to emerge: the use of the Internet as a global platform for machines and smart objects to communicate, compute and coordinate [1].

Computation and communication were two distinct concepts. They are now integrated in all large information systems and infrastructures. They have become a key factor in the spiral cycle of centralization and decentralization movement. People with the communication background tend to consider computing units to be the nodes of a network, while computer architects consider networks as the peripherals of computers for input and output purposes. The latest information infrastructure, the Internet of Things (IoT), cloud computing environments, ubiquitous networks, Linked Data Web, and autonomous decentralized systems, consider both computing and communication quantifiable resources that offer flexible levels of performance and quality on demand.

IoT will lead to major transformations in the field of information technology, and is considered to be the third wave of the information industry revolution after the invention of computer, Internet and the mobile communication network. It is the extension of Internet. Based on the standard and interoperable communication protocol, IoT connects almost any physical object with the Internet for information exchange and communication through sensors. Thus, IoT is a kind of network that can identify, locate, track, monitor and manage virtual or physical objects which connect to the Internet intelligently. Its basic characteristics include overall perception, reliable transmission and intelligent processing of information, and the most important is the information exchange between things and things or between humans and things [2].

Wireless Sensor Networks (WSNs) is a multi-hop autonomous network system formed by a large number of sensor nodes, which is deployed in monitoring areas to communicate with each other. It is an important technology for the IoT underlying network [3]. Since wireless communication, sensor technology, embedded applications and micro electronic technology have grown matured, the WSNs can obtain the required information at any time, any place and under any environmental condition. This lays the foundation for the development of IoT. WSNs possess multiple technical advantages, including autonomy, fast deployment, high fault tolerance and strong concealment etc. These characteristics make WSNs ideal for defining target geo-locations on battlefield, physiological data collection, intelligent transportation systems, as well as sea exploration and other fields [4]. 


\section{Literature Review}

Many scholars proposed different theoretic system architectures of IoT based on their research focuses. However, research on architecture of IoT is at the early stage and there are limited successful applications. It is difficult to make an objective evaluation of these different varieties of system architecture.

The paper [5] constructs its IoT architecture based on cloud computing. The autonomous management system tightly integrated the following services with the Aneka framework: Accounting, Monitoring, Profiling, Scheduling, and Dynamic Provisioning. Accounting, Monitoring, and Profiling will feed the sensors of the autonomic manager, while the managers' effectors will control Scheduling and Dynamic Provisioning. Logically, the two components that will take most advantages of the introduction of autonomic features in Aneka are the Application Scheduler and the Dynamic Resource Provisioning.

A system of smart objects that are interconnected digitally needs to be equipped with suitable interfaces, as the applications need to communicate by directives and compute effectively in order to leverage the data from multiple sources. The taxonomy of these main research areas and related topics are relevant to IoT [1]. The system architecture diagram presents a distributed intelligence.

Though many authors have put forward their own system architecture diagrams, all low-level networks were based on RFID technology [4, 6, 7]. However, these implementations for IoT via RFID were restricted due to its limited storage and one-directional communication. The IoT is defined as a huge network, which is a mixture of Internet, and requires real-time information of any necessary monitored, connected and interactive objects and processes, such as sound, light, heat, electricity, mechanics, chemistry, biology and location, all of which are collected by varieties of sensors and equipment. The aim is to achieve "things and things", "things and people" communication. All of the items being connected to the network are for easy identification, management and control. It should not only be confined to only functionally limited RFID implementations.

\section{The Improved Embedded System}

A cyber-physical system (CPS) is a system featuring a tight coordination between the system's computational and physical elements. Today, a precursor generation of cyber-physical systems can be found in many 
diverse areas, such as aerospace, auto industry, chemical processes, civil infrastructure, energy, healthcare, manufacturing, transportation, entertainment, and consumer appliances, etc. This generation is often referred to as embedded systems, in which the emphasis tends to be on the computational elements, instead of close link between the computational and physical elements.

A robot is a perfect example of intelligent physical devices. It is usually a system, which conveys a sense that it has intent or agency of its own by its performances or movements. We will study the three aspects of the system: structure, interface, and behaviour.

To prove the concepts, we have implemented a number of robots in the past few years, as shown in Figure 1. The architecture of IoT can be divided into three layers: Perception layer, Network layer and Application layer.

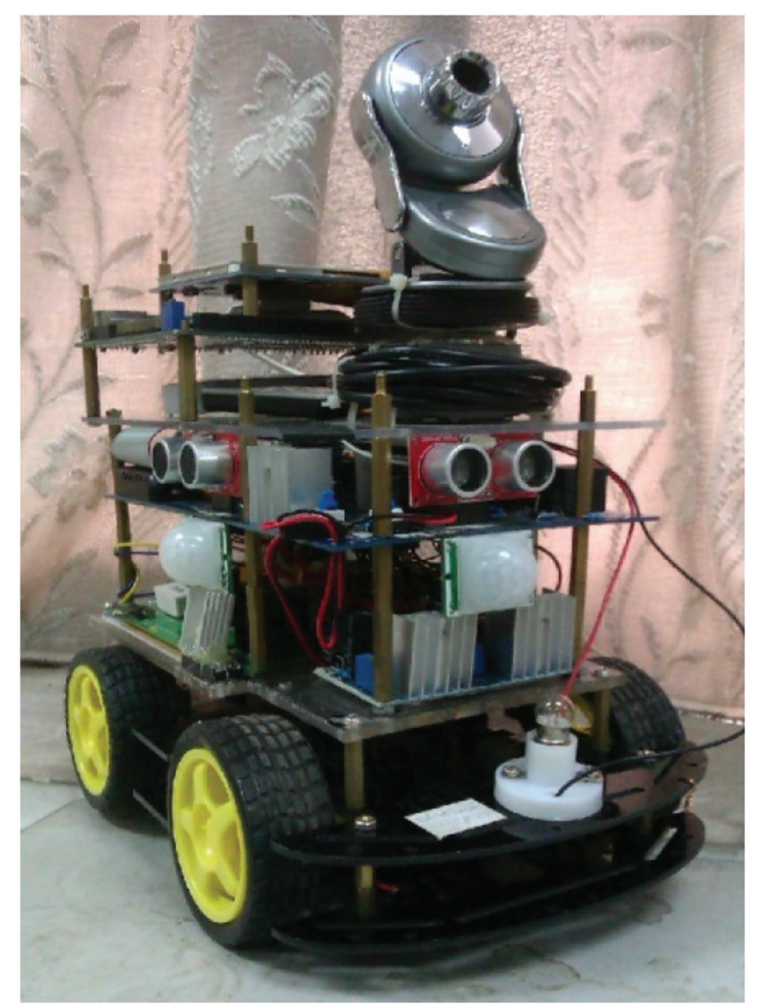

Figure 1 The intelligent robot. 


\subsection{System Architecture}

The System architecture is shown in Figure 2.

\subsubsection{Perception layer}

At first, we would focus on the perception of the outside world information. The Robot is based on MCU, which contains many sensors that can detect temperature, humidity, brightness, barrier, etc. We installed a wireless module in the Robot to achieve remote control by any designated computer. This method of controlling the Robot is basically the same as that of the toy car, but it is able to achieve two-way exchanges of data. In addition, the test data generated from the controlled object are transmitted to computer.

\subsubsection{Network layer}

The next important goal is to connect the Robot with a network. Most mobile devices using wireless communication can achieve bi-directional data exchange, but only for point-to-point connection. We use the embedded system as a core component in the Robot, so that mobile devices can be set as a Web server and set up a Wi-Fi hotspot. So we could control the Robot and obtain a variety of information by smart cell phones or computers through the Internet.

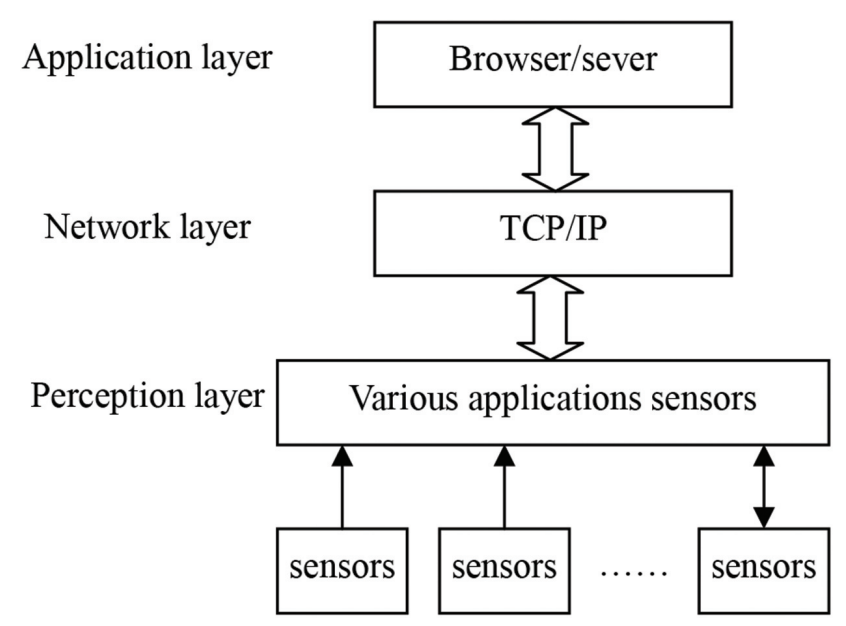

Figure 2 System architecture. 


\subsubsection{Application layer}

At first, we developed the client software to achieve a two-way exchange of data. The system works on a Client/Server mode.

Up until this stage, it is not yet very convenient to use although we have achieved physical elements connecting to the network. Now, the Robot is used as a server and the control of the Robot can be achieved by Wi-Fi enabled computers or smart phones' browsers other than client software. The system is now worked on a Browser/Server.

\subsection{System Structure and Function}

\subsubsection{Hardware system design}

The embedded system and a TQ2440 motherboard are used. The major components include:

the 400MHz ARM9 S3C2240 processor;

USB and I2C Communications Modules. The USB is applied to camera and $\mathrm{Wi}-\mathrm{Fi}$, and $\mathrm{I} 2 \mathrm{C}$ is applied to digital sensors, including compass sensor, acceleration sensor, gyro sensor, temperature sensor and humidity sensor. Other sensors use general I/O to communicate with CPU, such as the sonar sensor, motion sensor, and thermo sensor. So the terminal can collect the following information, light intensity, speed, distance between barriers, body infrared emission, temperature, humidity, slope and forward direction. There are also motors and relays on the terminal. A step-motor is used to control the camera, while the relays are used to control a bulb. Service-oriented computing model is used here, where each device is wrapped as a WSDL Web service, as shown in Figure 3. The Web services enable the Web accesses to the devices from the browser.

In our implementation, the software, standard interfaces and the Web capacity are the main considerations. Thus, A version of the Linux operation system has been implemented.

Programming languages: The $\mathrm{C}$ programming language is used to program the drivers, the services and the applications.

\subsubsection{Software system design}

Before describing the design of the software system, we first depict the information flow. The system flow chart of the system is shown in Figure 4.

The information gathered by sensors is transmitted to the S3C2240. The S3C2240 sends the information to the executive devices to control them to run, and shows the information on the user interface. 


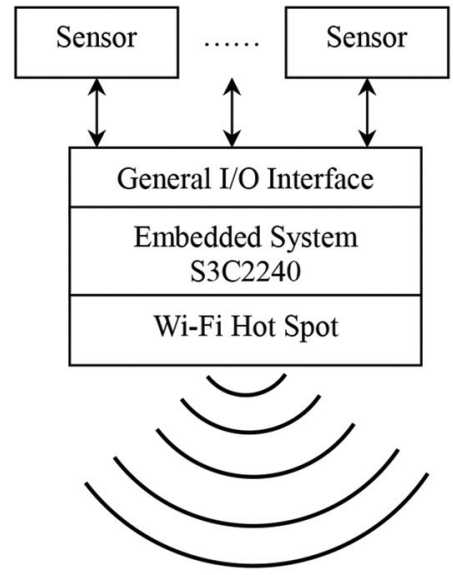

Figure 3 Block diagram of IoT.

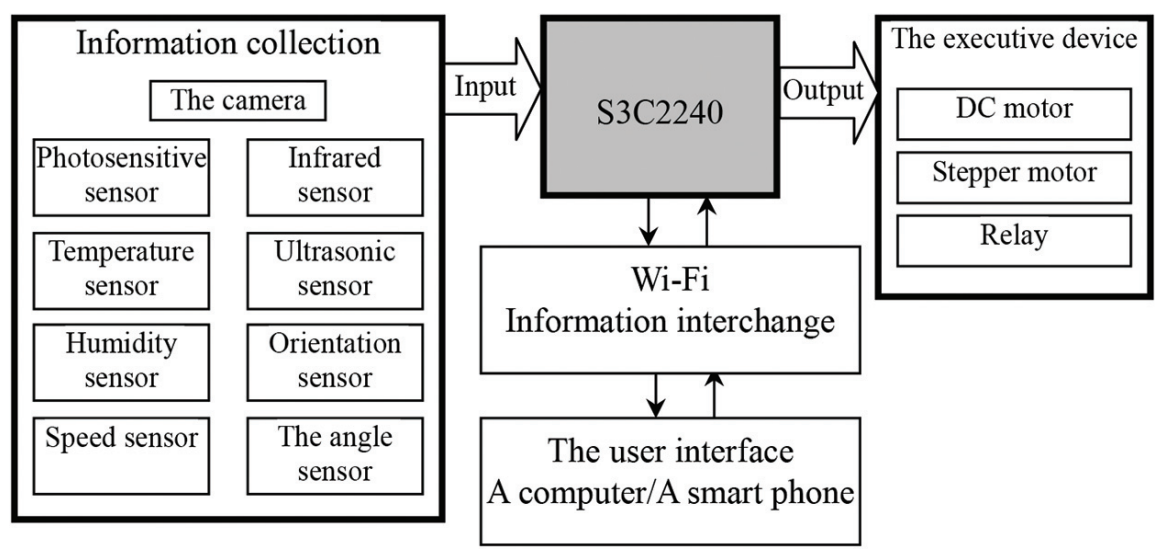

Figure 4 Information flow diagram.

The executive devices can also be controlled by the user through the user interface.

The software architecture of the operating system, the so called local system that runs on the intelligent robot local system installed on the car is shown in Figure 5. There is a multi-threaded monitor and control process. The monitor and control process achieve functions and monitor all the sensors loaded on the system and control all the controllers, just like dc motors. 


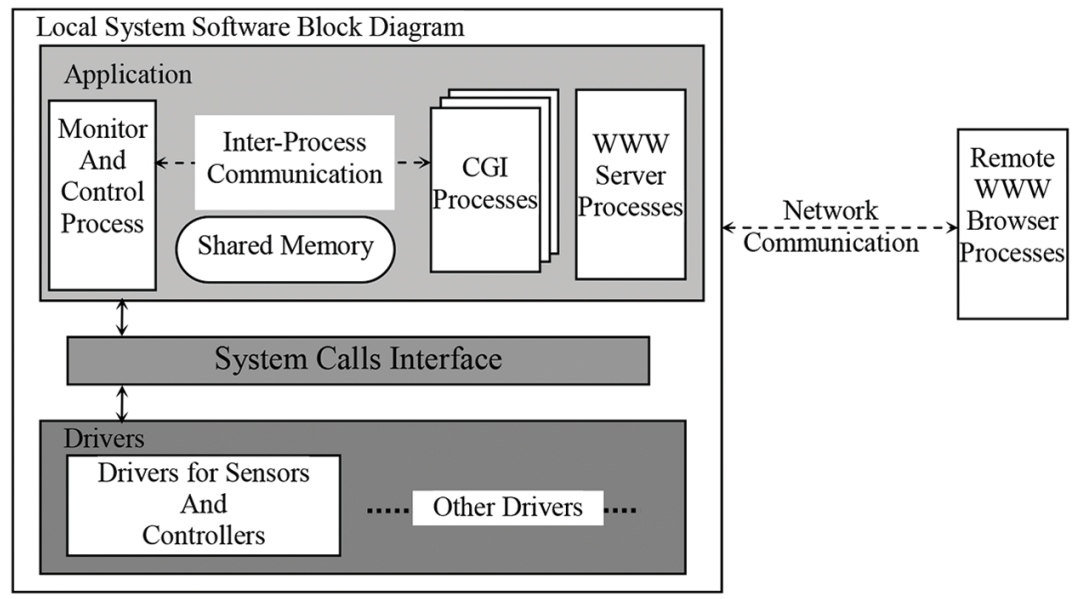

Figure 5 Block diagram of application software.

In order to provide services of data exchange with the remote WWW browser, we set up the local system with a WWW server which responds to requests submitted from the remote WWW browser with a mechanism called Common Gateway Interface (CGI). CGI processes act as a proxy; practically complete the tasks submitted from a remote browser and feed back the results.

An Inter-Process Communication approach called shared memory is used in the local system. Shared memory is a virtual memory space that can be accessed in a shared way for the processes running on the local system. It enables the monitor and control process to communicate with CGI processes.

\subsubsection{Description of the achieved functions}

Two kinds of functions have been achieved in our work: monitoring and controlling. On the client side, both functions are based on AJAX technology.

AJAX is a group of interrelated web development techniques used on the client-side to create asynchronous web applications. With Ajax, web applications can send data to, and retrieve data from, a server asynchronously (in the background) without interfering with the display and behaviour of the existing web page. Data can be retrieved using the XMLHttpRequest object. Despite the name, the use of XML is not required, and the requests do not need to be asynchronous. 
The monitoring function gets the sensor data by accessing the sensors loaded on the local system, subsequently stores the data into a pointed shared memory. The process of cyber server acts like a CGI, which returns the sensor data by reading the pointed shared memory while receiving feedback requests from the remote WWW browser. Remote browser will create an asynchronous request object to submit requests and handle the return data by using the AJAX technology, and then refresh the elements shown on the HTML page with the DOM+ model. A browser screenshot is shown in Figure 6.

The controlling function makes a thread cyclically check the control flags which are stored in the specified shared memory. It takes the appropriate actions when a signal of control flag is detected. The WWW server activates a CGI process, then sets the control flags stored in the pointed shared memory. The controlling functions operate the motors and relays according to the control flags.

\section{Discussion of the Experiment}

The system allows any user to use a computer or a smart phone to achieve the controlling of the robot through a web browser. As long as the user logins in the Robot server, the browser will display the interface shown in Figure 6, and

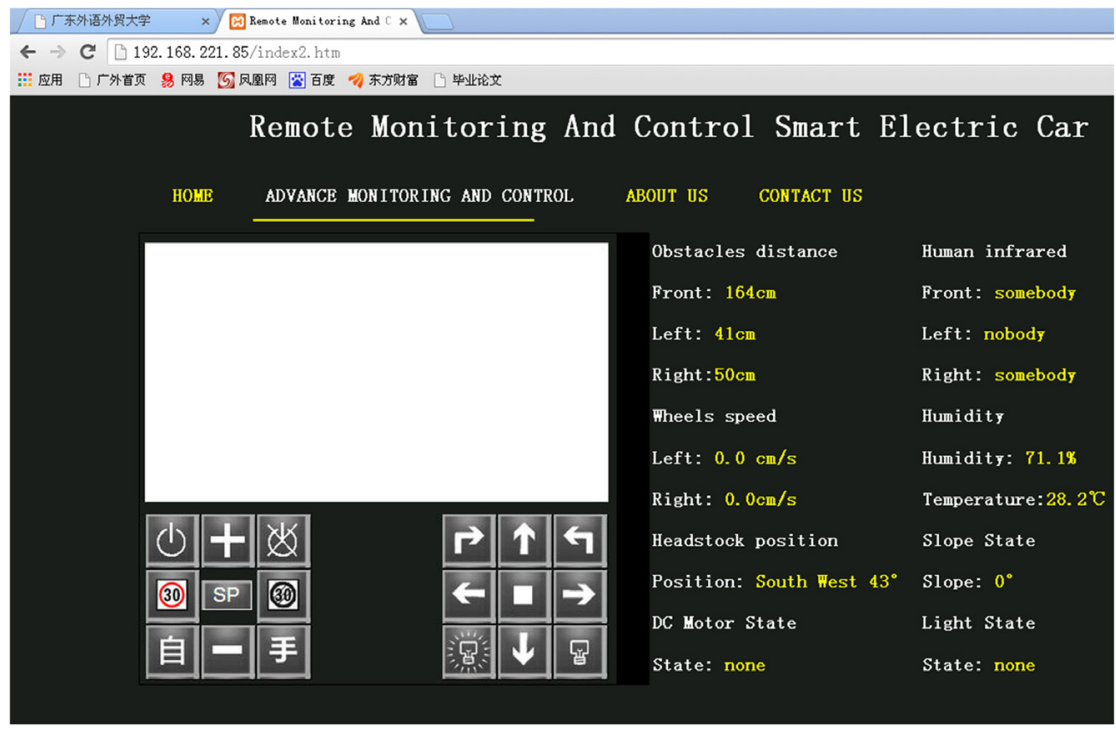

Figure 6 Browser screenshots. 
to implement the two-way transmission of data. This interface can display a variety of data that are detected by the Robot, and the Robot can also be controlled by the control buttons on the interface to implement real-time control.

The difference between the robot and other wireless control devices is that the control core is used to control and set the Web server to make the mobile device connect to the Internet. This may be called a mobile IoT.

The experiment situation is described below:

- Equipment Application

The device can be used to detect some circumstances, like the hazardous environment or in a limited space etc, including temperature, brightness, humidity, slope, orientation, and personnel information, and the video can be transmitted from the Robot. The lighting equipment will be on automatically to ensure shooting video in a dark environment.

These functions can also be implemented with traditional wireless remote control equipment, but it must have a dedicated remote control. In this experiment, any intelligent device with a Wi-Fi can control the Robot.

- Operation Mode

Table 1 Test results

\begin{tabular}{|c|c|c|}
\hline Item & Accuracy & Description \\
\hline Distance to obstacles & $1 \mathrm{~cm}$ & $\begin{array}{l}\text { The robot can turn around } \\
\text { obstacles within } 10 \mathrm{~cm}\end{array}$ \\
\hline Distance to living creatures & $4 \mathrm{~cm}$ & $\begin{array}{l}\text { Living creatures such as human } \\
\text { bodies or animals }\end{array}$ \\
\hline Wheel Speed & $0.01 \mathrm{~cm} / \mathrm{s}$ & $\begin{array}{l}\text { The speeds of the left wheel and } \\
\text { the right wheel are measured } \\
\text { separately, while the accuracy is } \\
\text { relative to the diameter of the } \\
\text { wheels }\end{array}$ \\
\hline Humidity & $0.5 \%$ & \\
\hline Temperature & $0.1^{\circ} \mathrm{C}$ & \\
\hline Direction & $1^{\circ}$ & \\
\hline Gradient & $0.5^{\circ}$ & \\
\hline Brightness & & $\begin{array}{l}\text { The lights of the car can be } \\
\text { automatically controlled }\end{array}$ \\
\hline Video & & The video is not smooth enough \\
\hline
\end{tabular}


The manual control mode:

The Robot can be controlled to move forward, backward, turn left, turn right, accelerate, and decelerate.

The camera can be controlled to rotate by 360 degrees.

Automatic cruise mode:

The Robot is provided with an automatic obstacle avoidance function, it can automatically turn around obstacles. The cruise speed can be controlled by the operator.

- Problems

The use of the $400 \mathrm{MHz}$ ARM9 S3C2240 processor can implement various detection and control functions. However, it lacks image processing capability. Therefore, the video signal is not smooth enough.

Table 1 shows the results of our test.

\section{Conclusion}

IoT brings a new age for IT technologies and can elevate our lives and job to a more intelligent and modern stage. The research and application of component technologies and the new application mode of IoT such as sea computing can facilitate the IoT to much wider fields.

This paper discussed the technologies and systems related to the Internet of Intelligent Things. And a design based on the architecture, interface, and behaviour is studied. As it is still a new and open domain, and much of the research still needs to be done. In our work, we didn't use a unified standard but a variety of sensor interface, which is more difficult to design. In order to adapt to the rapid development of the IoT needs, a variety of sensors interface with industry standards could be developed.

\section{Acknowledgements}

This work is supported by Guangdong Provincial Science and technology projects \#2013B040401015

\section{References}

[1] D. Miorandi, S. Sicari, F. D. Pellegrini, I. Chlamtac, Internet of things: Vision, applications and research challenges, Ad Hoc Networks, 2012, Vol. 10, No. 7, pp. 1497-1516. 
[2] Hu Yong-Li, Sun Yan-Feng, Yin Bao-Cai. Information Sensing and Interaction Technology in Internet of Things, Chinese Journal of Computers. 2012, Vol. 35, No. 6, pp. 1147-1163 (in Chinese).

[3] Liu Q. Huang X H. and Leng S P. Deployment strategy of wireless sensor networks for Internet of Things [J]. China Communications, 2011, Vol. 8, No. 8, pp. 111-120.

[4] Qian Zhi-hong, Wang Yi-jun, Internet of Things-oriented Wireless Sensor Networks Review, Journal of Electronics \& Information Technology. 2013, Vol. 35, No. 2, pp. 215-227 (in Chinese).

[5] J. Gubbi, R. Buyya, S. Marusic, M. Palaniswami, Internet of Things (IoT): A vision, architectural elements, and future directions. Future Generation Computer Systems. 2013, No. 29, pp. 1645-1660.

[6] K. Gama, L. Touseau, D. Donsez, Combining heterogeneous service technologies for building an Internet of Things middleware. Computer Communications. 2012, No. 35, pp. 405-417.

[7] X. Xu, T. Chen, M. Minami, Intelligent fault prediction system based on Internet of things, Computers and Mathematics with Applications. 2012, No. 64, pp. 833-899.

\section{Biographies}

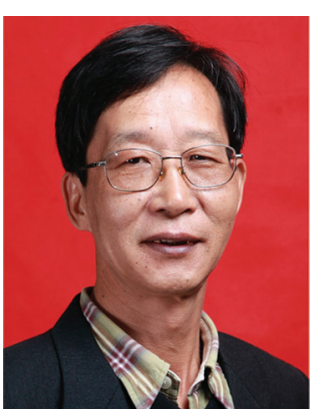

J. Wang associate professor. The research interest is information system and embedded system. Now he works in Guangdong University of Foreign Studies. 


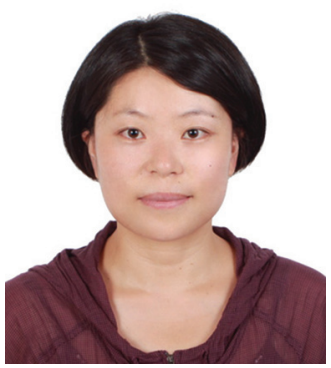

J. Zhang associate professor. The major field of study is embedded system and pattern classification. Her current job is teaching and works in Guangdong University of Foreign Studies.

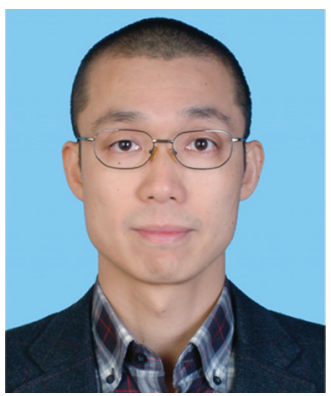

M. Yao associate professor, MSc., Research interest is natural language processing and software engineering. Now he works in Guangdong University of Foreign Studies.

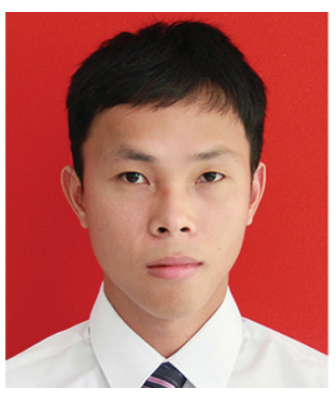

S. Li graduated from Guangdong University of Foreign Studies and now works at Guangzhou Shiyuan Electronic Technology Co., LTD. He has been engaged in embedded system software development, specializes in system architecture. 


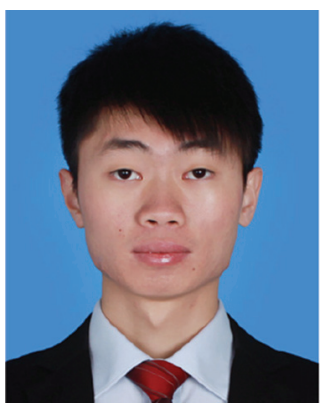

J. Li graduated from Guangdong University of Foreign Studies. Now take office in vipshop (China) Co., Ltd., mainly engaged in information system planning and design of monitoring system, monitoring object covers infrastructure, network, system, program and data. 